\title{
Ischemic Preconditioning and Acute Recovery of Performance in Rugby Union Players
}

\section{()(1) $\circledast \Theta$}

\author{
Authors
}

Cintia A. Garcia ${ }^{1}$, Gustavo Ribeiro da Mota' ${ }^{1}$, Anthony Scott Leicht ${ }^{2}$, Moacir Marocolo ${ }^{3}$

\author{
Affiliations \\ 1 Human Performance and Sport Research Group, \\ Department of Sport Sciences/Institute of Health \\ Sciences, Federal University of Triangulo Mineiro, \\ UBERABA, Brazil \\ 2 Sport and Exercise Science, James Cook University, \\ Townsville, Australia \\ 3 Department of Physiology, Federal University of Juiz de \\ Fora, Institute of Biological Sciences, Juiz de Fora, Brazil
}

\section{Key words}

ischemia, reperfusion, team sports, athletes

$\begin{array}{ll}\text { received } & 30.01 .2017 \\ \text { revised } & 03.04 .2017 \\ \text { accepted } & 01.05 .2017\end{array}$

\section{Bibliography}

DOI https://doi.org/10.1055/s-0043-111082

Sports Medicine International Open 2017; 1: E107-E112

(c) Georg Thieme Verlag KG Stuttgart · New York

ISSN 2367-1890

\section{Correspondence}

Gustavo Ribeiro da Mota, PhD

Human Performance and Sport Research Group

Department of Sport Sciences/Institute of Health Sciences

Federal University of Triangulo Mineiro

Av. Frei Paulino, 30, UBERABA, Brazil

\author{
38025-180, UBERABA \\ Brazil \\ Tel.: + 34/3318 5931, Fax: + 34/33185931 \\ grmotta@gmail.com
}

\begin{abstract}
Ischemic preconditioning has been used as a training and/or pre-competition strategy; however its use for post-exercise recovery is still unclear. This study aimed to evaluate the impact of ischemic preconditioning on performance and recovery ratings following a simulated match in sub-elite rugby players. Following baseline measures, male players $(n=8)$ performed a $40 \mathrm{~min}$, rugby-specific exercise protocol followed by an intervention: $21 \mathrm{~min}$ of ischemic preconditioning $(3 \times 5 \mathrm{~min}$ occlusion at $220 \mathrm{mmHg}$ with $2 \mathrm{~min}$ reperfusion at $0 \mathrm{mmHg}$ ) or passive rest (control) on 2 separate days. An agility T-test, a single vertical countermovement jump and $30 \mathrm{~s}$ of continuous vertical jumps were performed at baseline $(-24 \mathrm{~h})$, immediately after exercise, and immediately after the intervention. The rugby-specific exercise protocol induced similar mean heart rates ( $158.3 \pm 18.0$ vs. $158.7 \pm 16.0 \mathrm{bpm}$ ) and perceived exertion levels $(8.2 \pm 0.9$ vs. $8.0 \pm 1.0)$ for both trials with all recovery performance measures and rating of recovery $(13.9 \pm 1.4 \mathrm{vs}$. $13.6 \pm 1.6)$ similar between ischemic preconditioning and control trials (best $p=0.385)$. We conclude that the use of ischemic preconditioning does not improve recovery acutely $(\sim 1 \mathrm{~h})$ including specific variables related to rugby performance in amateur rugby union players.
\end{abstract}

\section{Introduction}

Ischemic preconditioning (IPC) is a procedure that induces tissue ischemia via circumferential compression of limb(s) followed by reperfusion in a repeated, cyclic manner $[18,30]$. This occlusive practice has generated considerable interest recently, with IPC reported to enhance exercise performance [18]. The mechanisms for IPC as an ergogenic aid are unclear, but may be related to local hyperemia inducing greater tissue oxygenation [24], enhanced mitochondrial function [1] and acceleration of muscle deoxygenation dynamics during moderate-intensity exercise [23]. Despite these potential beneficial effects during exercise, there are doubts about the efficacy of IPC for human performance [30], and due to the high heterogeneity of results (i.e., IPC responders and non-responders), authors suggest investigating the individual phenotype [18].

Several authors have attempted to demonstrate the beneficial effects of IPC on exercise performance $[7,10,15,20,30]$. For example, 10 strength-trained men who performed 5 sets of 5 maximal voluntary knee extensions of the right leg on an isokinetic dynamometer, preceded by either IPC of the right lower limb $(3 \times 5 \mathrm{~min}$ compression $/ 5 \mathrm{~min}$ reperfusion cycles at $200 \mathrm{~mm} \mathrm{Hg})$ or sham $(20 \mathrm{~mm} \mathrm{Hg})$, increased muscle perfusion and $\mathrm{O}_{2}$ uptake, conducive to higher repeated force capacity [31]. In a randomized, crossover study, 13 healthy men improved their $5 \mathrm{~km}$ running time trial performance after IPC, compared with a control intervention, 
accompanied by lower blood lactate concentrations at submaximal exercise workloads [2].

On the other hand, some authors suggest that IPC does not exceed the placebo effect and others that CPI has no effect on performance improvement $[33,36]$.

Despite some studies that report beneficial effects of IPC on simple actions and discrete exercise (e.g., running, swimming and cycling) $[18,30]$, little attention has been given to IPC use for team sport participants, and even less for IPC as a recovery modality. Proper recovery interventions are crucial for human performance and studies have attempted to find them [11,34]. To our knowledge, only 2 studies have investigated the effectiveness of IPC as a recovery intervention $[3,5]$. One study demonstrated no positive effects from IPC after a strenuous bout of eccentric exercise (3 sets of 100 repetitions), at 24,48 and $72 \mathrm{~h}$ post-exercise compared with a non-IPC (control) recovery period [5]. The other reported a beneficial effect of IPC on repeated sprint ability and jump height performances but only at $24 \mathrm{~h}$ after the intervention [3]. Although these study designs were interesting, the exercise bout examined was atypical in practical terms (i.e., involved a high volume of eccentric actions) with the recovery period also potentially too long for some athletes during sporting competition. For instance, during an amateur "sevens" rugby union tournament, matches commonly occur over a period of 2 to $3 \mathrm{~d}$ with only $\sim \mathrm{h}$ of rest between matches. Consequently, implementation of treatment between matches to improve recovery and enhance performance would be highly advantageous. Given that IPC induces local hyperemia, potentially higher tissue oxygenation [24], and enhanced mitochondrial function [1], IPC may provide a novel means to enhance recovery and subsequent performance within a short timeframe (i.e., $\sim 1 \mathrm{~h}$ ), similar to that during competitive tournaments. Thus, the aim of this study was to evaluate the effectiveness of IPC on acute recovery and performance in rugby union players.

\section{Materials and Methods}

\section{Participants}

Eight male, amateur rugby union players $(24 \pm 4 \mathrm{yrs} ; 179 \pm 5 \mathrm{~cm}$; $88 \pm 9 \mathrm{~kg}$ ) competing at the state level, participated as volunteers. All participants were informed about the experimental procedures and gave written informed consent prior to participation in accordance with approval by the local Ethical Committee for Human Experiments and meet ethical standards in sport and exercise science research [16]. Prior to the study, all players had undergone at least 6 months of rugby-specific training, with the study taking place during the pre-competition phase of the season. None of the players reported any cardiovascular, pulmonary, metabolic diseases, musculoskeletal injuries, skin or gastrointestinal infections. Further, none reported to have used anabolic steroids or drugs that may have affected physical performance, and none were taking any anti-inflammatory drugs for at least one week before the study.

All players were nonsmokers and were instructed to avoid any consumption of food or drinks in the $3 \mathrm{~h}$ prior to testing and to refrain from alcohol consumption and exercise in the $24 \mathrm{~h}$ prior to the testing session. Caffeine ingestion was limited to coffee at
06:30-09:00 with the rugby-specific exercise protocol and performance tests beginning at $16: 00$.

\section{Experimental protocol}

A randomized crossover design was used to investigate the effect of IPC on performance during the acute recovery $(\sim 1 \mathrm{~h})$ period following a rugby-specific exercise protocol ( $\vee$ Fig. 1 ). Beyond the anthropometric measures and familiarization with all physiological, neuromuscular, and perceptual procedures previously performed, the players performed 2 complete trials with 1 -week between trials. The trials differed only in the recovery strategy (i.e., $21 \mathrm{~min}$ of IPC or passive rest - control). The rugby-specific exercise protocol and performance tests took place at the same time of day (starting at 16:00) to minimize any confounding effects of daily biorhythms. Players completed a set of performance tests followed by the first exercise protocol and post-exercise assessments. The performance tests ( $T$-test, single vertical countermovement jump and $30 \mathrm{~s}$ continuous vertical jumps test), were conducted 6 times during the study: i) at baseline (separate day); ii) immediately after the rugby-specific exercise protocol; and iii) after the intervention (IPC or control) during each of the 2 testing sessions, one week apart ( $\vee$ Fig. 1). These tests were previously employed in studies involving rugby union players and reported to exhibit high validity and reliability for rugby union players $[9,19,38]$.

\section{Rugby-specific exercise protocol}

In an indoor gymnasium $\left(21-24^{\circ} \mathrm{C}, 50-60 \%\right.$ relative humidity), after a 7 min warm-up (i.e., 5 min of slow jogging and 2 min of dynamic and static stretching), the players completed the rugby-specific exercise protocol that simulated the physical and skill demands of a rugby union match $[14,35]$. The protocol simulated a match half $(40 \mathrm{~min})$ and consisted of 8 stations performed in the following order ( 5 min each with $30 \mathrm{~s}$ of passive rest between them): i) jumps; ii) skill passing; iii) position scrum with member alternation; iv) slalom agility sprints; v) rest and hydration; vi) dragon walks; vii) slalom agility sprints; and viii) $20 \mathrm{~m}$ sprint. All players received similar verbal encouragement by an independent evaluator, who did not know the experimental condition, and completed the protocol as a rotational circuit (same order of tests, in sequence).

To ensure that the internal intensity was similar across the 2 trials, all players wore a Polar RS800CX ${ }^{\circledR}$ heart rate (HR) monitor (Polar Electro Oy, Kempele, Finland) during each trial with rating of perceived effort ([RPE], 0-10) [37] obtained at the end of each station. The HR was continuously assessed during the exercise protocol and analyzed as the average for all 8 stations. Similarly, analysis of RPE utilized the mean rating across all stations.

\section{Performance Tests}

\section{T-test}

T-test was performed at baseline (separate day), $1 \mathrm{~min}$ after the rugby-specific exercise protocol and $1 \mathrm{~min}$ the intervention (IPC or control). The players performed the agility test as fast as possible twice, separated by $1 \mathrm{~min}$, as per the standardized protocol [32]. Briefly, players ran $9.14 \mathrm{~m}$ followed by side stepping to the left $(4.57 \mathrm{~m})$, side-stepping to the right $(9.14 \mathrm{~m})$, side-stepping to the left $(4.57 \mathrm{~m})$ and backward running $(9.14 \mathrm{~m})$ to the start/finish line. 


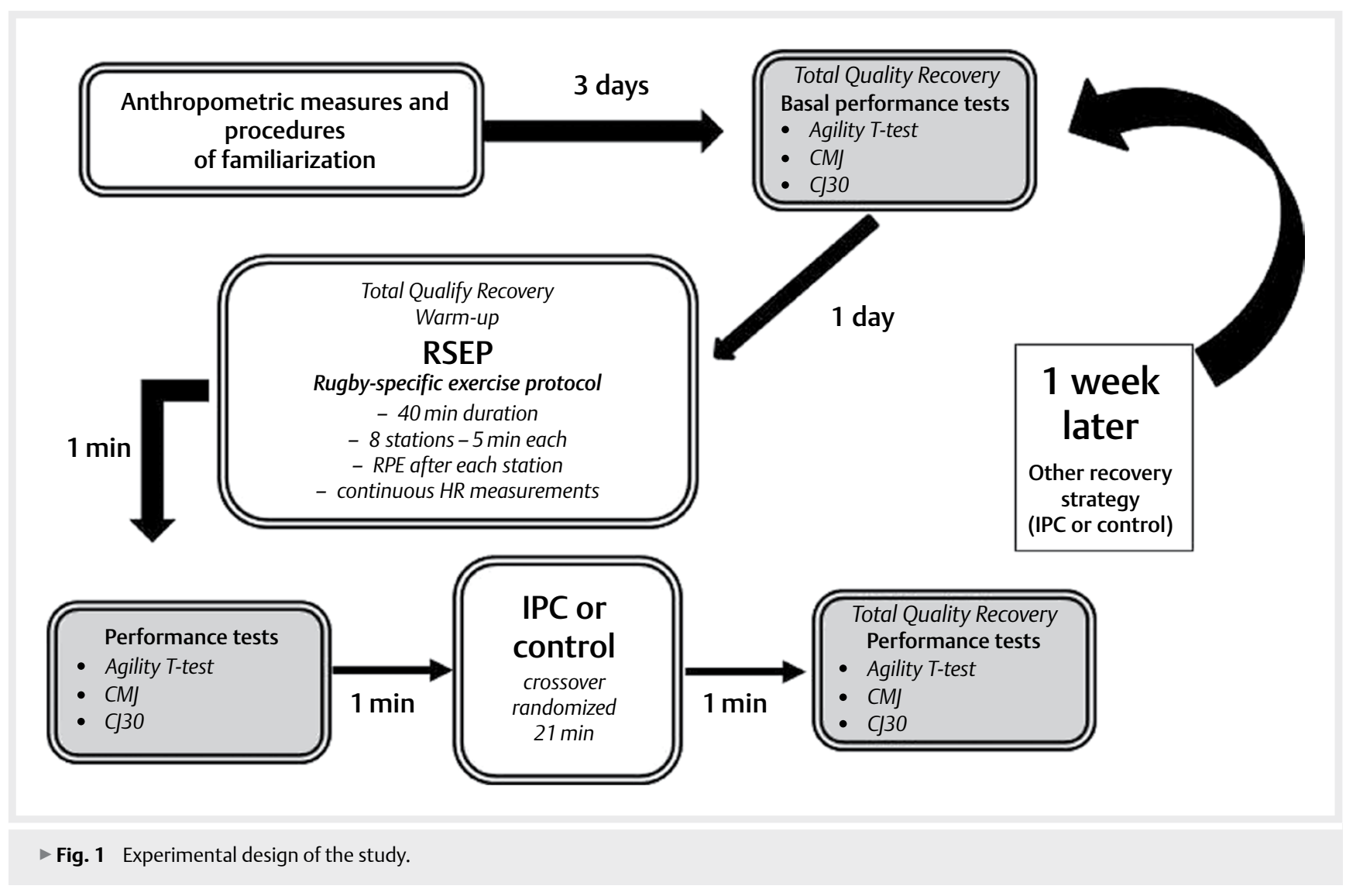

Time of completion was manually recorded by 3 trained assessors using chronometers (SEIKO S141-300, Japan) and averaged. Assessors were blinded with regards to the recovery interventions, as previously suggested [8]. The T-test has been reported to exhibit high validity and reliability across trials with a reliability coefficient of 0.98 [32]. To ensure minimal measurement errors, inter-assessor reliability in the current study was examined using an intra-class correlation coefficient (ICC) with a mean ICC value of 0.878 (95\% $\mathrm{Cl}=0.836-0.917 ; \mathrm{p}=0.033)$ calculated.

\section{Countermovement jump test (CMJ)}

All players performed the CMJ on a contact mat system (Just Jump, Probotics, Inc., Huntsville, USA) with their hands kept on their hips for the entire jump [25] after the T-test. 3 standardized jumps ( $90^{\circ}$ knee flexion) were completed with $30 \mathrm{~s}$ rest between jumps. Jump flight time was measured via the contact mat and jump height calculated using the participant's mass [25]. The best CM] height was considered for analysis. The CMJ has been employed as a practical and commonly used valid test of leg power with good intra-day (coefficient of variation $-C V=5.2 \%)$ and inter-day $(C V=5.0 \%)$ jump height reliability [6].

\section{0 s continuous jump test (CJ30)}

The CJ30 test was performed after CJM and consisted of the performance of maximal, continuous vertical jumps during $30 \mathrm{~s}$ using the same contact mat system described above. All players were instructed to maintain a standardized jump technique where the torso was as vertical as possible, hands crossed at their chests and a knee joint flexion angle of $\sim 90^{\circ}$ at the bottom of the jump. Jump height was calculated as described above with the average jump height of all jumps during the 30 s period used for analysis. The CJ30 has been reported to be a reliable indicator of muscular endurance and performance [9].

\section{Recovery strategies - ischemic preconditioning (IPC) protocol or control}

The $21 \mathrm{~min}$ IPC protocol consisted of 3 cycles of 5 min occlusion at $220 \mathrm{mmHg}$ using a $96 \mathrm{~cm} \times 13 \mathrm{~cm}$ pneumatic cuff applied around the sub-inguinal region of the upper thighs (Komprimeter Riester ${ }^{\circledR}$, Jungingen, Germany) alternated with 2 min of reperfusion at $0 \mathrm{mmHg}$ [3]. The applied pressure and cuff width were in accordance with that used in previous studies [28] to ensure that blood flow was occluded during the intervention. The occlusion and reperfusion phases were conducted alternately between the thighs (i.e., each thigh was occluded for 3 cycles of occlusion/reperfusion) with participants remaining seated during the entire $21 \mathrm{~min}$. The effectiveness of occlusion during the IPC session was confirmed by auscultation of the arteries around the ankle [28] during the start of each occlusion/reperfusion phase. No cuff was applied to the thighs during the control protocol and participants sat passively for 21 min.

\section{Rating of recovery}

At baseline, before and after the 2 trials of rugby-specific exercise protocol (IPC or control), the players reported their rating of recov- 
ery using a total quality recovery scale ranging from 6 ( very, very poor recovery) to 20 ( very, very good recovery) [22].

\section{Statistical analysis}

The Shapiro-Wilk test was employed to verify the normal distribution of the data. Comparisons of data related to the rugby-specific exercise protocol (HR, RPE) were conducted using paired T-tests (normal distribution) or Wilcoxon matched-pairs signed-rank tests (non-normal distribution). Examination of variables between interventions (IPC vs. control) at each time point was carried out via Wilcoxon matched-pairs signed-rank tests with a Bonferroni correction for the number of comparisons. The significance level was set at 0.05 and all analyses were conducted using GraphPad ${ }^{\circledR}$ (Prism 6.0, San Diego, CA, USA).

\section{Results}

During the rugby-specific exercise protocol, mean $\mathrm{HR}$ $(158.3 \pm 18.0 \mathrm{bpm}$ vs. $158.7 \pm 16.0 \mathrm{bpm}, \mathrm{p}=0.96)$ and $\operatorname{RPE}(8.2 \pm 0.9$ vs. $8.0 \pm 1.0, p=0.69$ ) were similar prior to the IPC and control interventions, respectively. At baseline, the rating of recovery was similar for the IPC and control trials ( $18.5 \pm 0.5$ vs. $18.5 \pm 0.8$, $\mathrm{p}>0.05$ ) and similar to the values prior to the rugby-specific exercise protocol (IPC $=18.8 \pm 0.7$; control $=18.6 \pm 0.5, p>0.05)$. Following the rugby-specific exercise protocol, the rating of recovery was similar for both trials $(\mathrm{IPC}=13.9 \pm 1.4$; control $=13.6 \pm 1.6$, $p>0.05$ ).

At each time point (i.e., baseline, post-rugby-specific exercise protocol and post-intervention), T-test and CMJ results were similar for the control and IPC conditions ( $\vee$ Fig. 2a,b). Likewise, CJ30 performance was comparable $(p>0.05)$ after both the IPC and the control interventions ( $\vee$ Fig. $2 c$ c).

\section{Discussion}

Our main finding was that IPC of the thigh muscles (i.e., alternate unilateral occlusion) did not improve the short-term recovery of performance or perceived recovery status for amateur rugby players following a simulated match. During the short intervals of rest (e.g., $\sim 1 \mathrm{~h}$ ) between matches throughout a competitive tournament (i.e., several matches in the same day), IPC may not be an effective means to enhance athlete recovery and subsequent performance.

Prior studies of IPC and long-term recovery have produced mixed results $[3,5]$ with the only positive effect reported being increased repeated sprint ability and jump height at $24 \mathrm{~h}$ after IPC [3].

The authors attributed the IPC-induced improvement to increased blood flow (i.e., reperfusion) and muscular oxygen utilization for a faster recovery of muscular function. However, blood flow and oxygen consumption were not assessed in that study and their mechanistic statements were speculative. In the current study, no measure of performance was different between IPC and control trials immediately and $1 \mathrm{~h}$ following a simulated match. This lack of IPC effect was similar to that reported following a strenuous bout of eccentric exercise over $72 \mathrm{~h} \mathrm{[5]}$ and provides further inconclusive evidence of the recovery benefits of IPC.
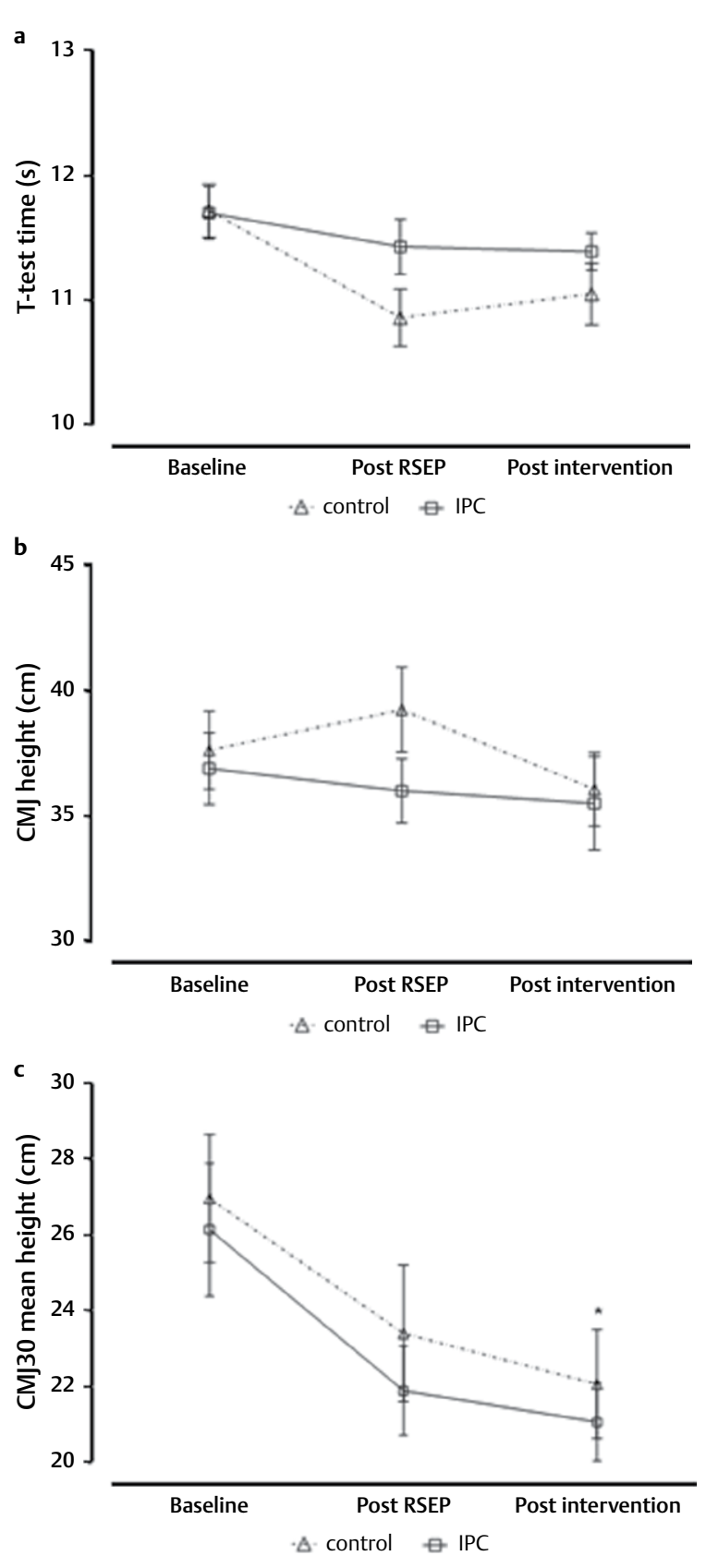

- Fig. 2 a T-test (agility), b countermovement jump, CMJ (leg power) and c continuous CMJ during 30-s (muscular endurance) at baseline, immediately after the rugby-specific exercise protocol (Post RSEP) and immediately after the intervention (Post Intervention) for the ischemic preconditioning (IPC) and passive rest (Control) trials. ${ }^{*}$ means $p<0.05$ vs. Baseline.

Because several matches in a single day are common during amateur rugby sevens tournaments and IPC has been reported to improve performance in exercise of predominantly lactic anaerobic and aerobic capacity [18], there is evidence suggesting a time-dependent effect of ischemic preconditioning, with the time window of the beneficial effect starting after about $2 \mathrm{~h}$ and lasting for at 
least $8 \mathrm{~h}$ after ischemic preconditioning [27]. However, the current results demonstrated no differences between IPC and control. Considering the time, logistics and pain involved with the IPC maneuver [26], passive recovery may be a more suitable recovery for these athletes during tournaments.

Importantly, the similar IPC and control results in the current study confirmed that IPC did not incur further decrements in performance. Despite not being beneficial for athletes in the short term, IPC did not harm athletes and/or reduce performance in the current study. Whether multiple applications of recovery IPC after many matches have the same, greater, or diminished effect has yet to be examined. With potentially increased muscular damage and fatigue occurring during repeated matches over a short time span such as a competitive tournament [21], the potential benefits of IPC may become more apparent. Whether these benefits involve improvement in muscle blood flow and oxygenation has yet to be validated with future studies encouraged to examine these parameters during tournament conditions. To our knowledge, no other study has examined recovery with IPC after a team sports simulated match within a short $(\sim 1 \mathrm{~h})$ or longer post-exercise time. Therefore, our study was unique in examining the post-exercise use of IPC for team sports and included a very controlled and robust design (i.e., counterbalanced crossover and randomized) that should be followed for future studies.

As indicated previously, similar performances were noted following IPC and control recovery procedures. These similarities were not due to altered performances of rugby-specific exercise protocol because the current HR responses and RPE were comparable between the 2 rugby-specific exercise protocols performed. Further, perception of recovery using the total quality recovery scale was equally lower after the simulated match for both interventions (IPC and control). Therefore, the 2 trials of rugby-specific exercise protocol were equally stressful, resulting in similar states for the recovery strategies. The cardiovascular response imposed on athletes ( $\mathrm{HR}$ of $158 \mathrm{bpm}, \sim 80 \% \mathrm{HR}_{\text {peak }}$ ) was similar to that experienced by amateur rugby players [13] and elite youth rugby league players (78.8-83\% HR $\mathrm{H}_{\text {peak }}$ ) [37]. Additionally, the rating of perceived effort (RPE) imposed on athletes ( $~ 8$ out of 10 ) was in line with comparable studies of rugby athletes [37]. Therefore, the current responses of $\mathrm{HR}$, in absolute and relative terms, as well as RPE, were in accordance with prior studies of rugby [35] and further highlight the ecological validity of the rugby-specific exercise protocol.

It is important that some limitations of the present study be documented. The absence of a sham/placebo trial could have affected results, because a potential placebo effect of IPC has been reported for some performance variables [29, 30]. A placebo effect may result from the interaction between psychological and physiological variables [4], however, this was unlikely given that no positive effects of IPC on performance were identified in the current study and the rate of recovery (i.e., a subjective measure that involves psychological factors) was also unaffected by IPC.

Secondly, the current study was limited to a small sample size. Previous studies examining IPC have reported beneficial effects of IPC with a similar sample size [12]. Further, we incorporated nonparametric analyses in the current study to minimize any impact of sample size. Finally, sub-elite players were examined in the current study with results possibly not translatable to elite athletes.
However, IPC interventions have promoted similar effects in both amateur [12] and highly trained cyclists [17] with future studies needed to identify possible unique IPC responses based on training status.

\section{Conclusions}

IPC does not improve recovery of performance acutely (within $1 \mathrm{~h}$ ) for sub-elite rugby players following a rugby-specific exercise protocol. The use of IPC between closely scheduled matches performed on the same day may therefore be of minimal benefit with other recovery strategies likely to be more advantageous for enhancing performance. The match and recovery benefits of IPC use during tournaments with greater between-match intervals $(\sim 3 \mathrm{~h})$ remains to be clarified.

\section{Acknowledgements}

Financial support for the author Gustavo R da Mota from The State Funding Agency of Minas Gerais - FAPEMIG (process ETC-00183-16).

\section{Conflict of Interest}

The authors declare that they have no conflict of interest.

\section{References}

[1] Andreas M, Schmid Al, Keilani M, Doberer D, Bartko J, Crevenna R, Moser E, Wolzt M. Effect of ischemic preconditioning in skeletal muscle measured by functional magnetic resonance imaging and spectroscopy: A randomized crossover trial. J Cardiovasc Magn Reson 2011; 13: 32

[2] Bailey TG, Jones H, Gregson W, Atkinson G, Cable NT, Thijssen DH. Effect of ischemic preconditioning on lactate accumulation and running performance. Med Sci Sports Exerc 2012; 44: 2084-2089

[3] Beaven CM, Cook C], Kilduff L, Drawer S, Gill N. Intermittent lower-limb occlusion enhances recovery after strenuous exercise. Appl Physiol Nutr Metab 2012; 37: 1132-1139

[4] Beedie C], Foad A]. The placebo effect in sports performance: A brief review. Sports Med 2009; 39: 313-329

[5] Cochrane D], Booker HR, Mundel T, Barnes M]. Does intermittent pneumatic leg compression enhance muscle recovery after strenuous eccentric exercise? Int J Sports Med 2013; 34: 969-974

[6] Cormack S], Newton RU, McGuigan MR, Doyle TL. Reliability of measures obtained during single and repeated countermovement jumps. Int J Sports Physiol Perform 2008; 3: 131-144

[7] Crisafulli A, Tangianu F, Tocco F, Concu A, Mameli O, Mulliri G, Caria MA. Ischemic preconditioning of the muscle improves maximal exercise performance but not maximal oxygen uptake in humans. J Appl Physiol 2011; 111: 530-536

[8] da Mota GR, Marocolo M. The effects of ischemic preconditioning on human exercise performance: A counterpoint. Sports Med 2016; 46: 1575-1576

[9] Dal Pupo J, Gheller RG, Dias JA, Rodacki AL, Moro AR, Santos SG. Reliability and validity of the 30-s continuous jump test for anaerobic fitness evaluation. J Sci Med Sport 2013, doi:10.1016/j. jsams.2013.09.007 
[10] de Groot PC, Thijssen DH, Sanchez M, Ellenkamp R, Hopman MT. Ischemic preconditioning improves maximal performance in humans. Eur J Appl Physiol 2010; 108: 141-146

[11] De Nardi M, La Torre A, Barassi A, Ricci C, Banfi G. Effects of cold-water immersion and contrast-water therapy after training in young soccer players. J Sports Med Phys Fitness 2011; 51: 609-615

[12] Foster GP, Westerdahl DE, Foster LA, Hsu JV, Anholm JD. Ischemic preconditioning of the lower extremity attenuates the normal hypoxic increase in pulmonary artery systolic pressure. Respir Physiol Neurobiol 2011; 179: 248-253

[13] Gabbett T, King T, Jenkins D. Applied physiology of rugby league. Sports Med 2008; 38: 119-138

[14] Garcia CA, da Mota GR, Marocolo M. Cold water immersion is acutely detrimental but increases performance post- $12 \mathrm{~h}$ in rugby players. Int J Sports Med 2016; 37: 619-624

[15] Gibson N, Mahony B, Tracey C, Fawkner S, Murray A. Effect of ischemic preconditioning on repeated sprint ability in team sport athletes. J Sports Sci 2015; 33: 1182-1188

[16] Harriss D], Atkinson G. Ethical standards in sport and exercise science sesearch: 2016 update. Int J Sports Med 2015; 36: 1121-1124

[17] Hittinger EA, Maher JL, Nash MS, Perry AC, Signorile JF, Kressler J, Jacobs KA. Ischemic preconditioning does not improve peak exercise capacity at sea level or simulated high altitude in trained male cyclists. Appl Physiol Nutr Metab 2015; 40: 65-71

[18] Incognito AV, Burr JF, Millar PJ. The effects of ischemic preconditioning on human exercise performance. Sports Med 2016; 46: 531-544

[19] Jarvis S, Sullivan LO, Davies B, Wiltshire H, Baker JS. Interrelationships between measured running intensities and agility performance in subelite rugby union players. Res Sports Med 2009; 17: 217-230

[20] Jean-St-Michel E, Manlhiot C, Li J, Tropak M, Michelsen MM, Schmidt MR, McCrindle BW, Wells GD, Redington AN. Remote preconditioning improves maximal performance in highly trained athletes. Med Sci Sports Exerc 2011; 43: 1280-1286

[21] Johnston RD, Gibson NV, Twist C, Gabbett TJ, MacNay SA, MacFarlane NG. Physiological responses to an intensified period of rugby league competition. J Strength Cond Res 2013; 27: 643-654

[22] Kentta G, Hassmen P. Overtraining and recovery. A conceptual model. Sports Med 1998; 26: 1-16

[23] Kido K, Suga T, Tanaka D, Honjo T, Homma T, Fujita S, Hamaoka T, Isaka T. Ischemic preconditioning accelerates muscle deoxygenation dynamics and enhances exercise endurance during the work-to-work test. Physiol Rep 2015; 3: pii: e12395

[24] Kjeld T, Rasmussen MR, Jattu T, Nielsen HB, Secher NH. Ischemic preconditioning of one forearm enhances static and dynamic apnea. Med Sci Sports Exerc 2014; 46: 151-155
[25] Komi PV, Bosco C. Utilization of stored elastic energy in leg extensor muscles by men and women. Med Sci Sports 1978; 10: 261-265

[26] Lalonde F, Curnier DY. Can anaerobic performance be improved by remote ischemic preconditioning? J Strength Cond Res 2015; 29: 80-85

[27] Lisboa FD, Turnes T, Cruz RS, Raimundo JA, Pereira GS, Caputo F. The time dependence of the effect of ischemic preconditioning on successive sprint swimming performance. J Sci Med Sport 2016, doi:10.1016/j.jsams.2016.09.008

[28] Loenneke JP, Fahs CA, Rossow LM, Sherk VD, Thiebaud RS, Abe T, Bemben DA, Bemben MG. Effects of cuff width on arterial occlusion: Implications for blood flow restricted exercise. Eur J Appl Physiol 2012; 112: 2903-2912

[29] Marocolo M, da Mota GR, Pelegrini V, Appell Coriolano HJ. Are the beneficial effects of ischemic preconditioning on performance partly a placebo effect? Int J Sports Med 2015; 36: 822-825

[30] Marocolo M, da Mota GR, Simim MA, Appell Coriolano H]. Myths and facts about the effects of ischemic preconditioning on performance. Int J Sports Med 2016; 37: 87-96

[31] Paradis-Deschenes P, Joanisse DR, Billaut F. Ischemic preconditioning increases muscle perfusion, oxygen uptake, and force in strengthtrained athletes. Appl Physiol Nutr Metab 2016; 41: 938-944

[32] Pauole KMK, Garhammer J, Lacourse M, Rozenek R. Reliability and validity of the T-test as a measure of agility, leg power, and leg speed in college-aged men and women. J Strength Cond Res 2000; 14: 3

[33] Sabino-Carvalho JL, Lopes TR, Obeid-Freitas T, Ferreira TN, Succi JE, Silva AC, Silva BM. Effect of ischemic preconditioning on endurance performance does not surpass placebo. Med Sci Sports Exerc 2017; 49: 124-132

[34] Soultanakis HN, Nafpaktiitou D, Mandaloufa SM. Impact of cool and warm water immersion on 50-m sprint performance and lactate recovery in swimmers. J Sports Med Phys Fitness 2015; 55: 267-272

[35] Stuart GR, Hopkins WG, Cook C, Cairns SP. Multiple effects of caffeine on simulated high-intensity team-sport performance. Med Sci Sports Exerc 2005; 37: 1998-2005

[36] Tocco F, Marongiu E, Ghiani G, Sanna I, Palazzolo G, Olla S, Pusceddu M, Sanna P. Corona F, Concu A, Crisafulli A. Muscle ischemic preconditioning does not improve performance during self-paced exercise. Int J Sports Med 2015; 36: 9-15

[37] Waldron M, Worsfold PR, Twist C, Lamb K. A three-season comparison of match performances among selected and unselected elite youth rugby league players. J Sports Sci 2014; 32: 1110-1119

[38] Webb NP, Harris NK, Cronin JB, Walker C. The relative efficacy of three recovery modalities after professional rugby league matches. J Strength Cond Res 2013; 27: 2449-2455 